\title{
RELACIÓN ENTRE LIDERAZGO TRANSFORMACIONAL Y TRANSACCIONAL CON LA CONDUCTA DE COMPARTIR CONOCIMIENTO EN DOS EMPRESAS DE SERVICIOS
}

\author{
Marta Gisela Durán Gamba ${ }^{1 *}$, Delio Ignacio Castañeda Zapata ${ }^{1 * *}$ \\ ${ }^{1}$ Universidad Católica De Colombia
}

Recibido, octubre 6/2011

Concepto de evaluación, octubre 12/2012

Aceptado, agosto 15/2014
Referencia: Durán Gamba, M.G. \& Castañeda, D.I. (2015). Relación entre liderazgo transformacional y transaccional con la conducta de compartir conocimiento en dos empresas de servicios. Acta Colombiana de Psicología, 18(1), 135-147. DOI: 10.14718/ACP.2015.18.1.13

Resumen

\begin{abstract}
El objetivo fue describir la relación entre el liderazgo transformacional-transaccional del líder con la conducta de compartir conocimiento de los colaboradores. Utilizando un diseño descriptivo-correlacional con análisis regresional y un muestreo incidental, se valoraron 150 trabajadores de dos empresas de Bogotá: 14 líderes y 136 colaboradores. Los instrumentos aplicados fueron el Multifactor Leadership Questionarie de Bass \& Avolio adaptado por Vega \& Zavala (2004) y la escala de Compartir Conocimiento de Castañeda (2010). El análisis estadístico se realizó con el paquete estadístico IBM-SPSS STATISTIC 20,0. Entre los líderes se encontró que el 43\% se caracteriza por un liderazgo de baja efectividad (bajo nivel transformacional y transaccional) y el $40 \%$, por un liderazgo de rango completo (alto nivel transformacional y transaccional). En los colaboradores, el compartir conocimiento puntuó entre 4,3 y 5,4/6,0; sobresale compartir experiencias e ideas y, en menor nivel, compartir documentos y conocimientos. Desde la perspectiva de los jefes, el liderazgo transaccional correlacionó significativamente $(\mathrm{r}=0,63 ; \mathrm{p}=0,01)$ con compartir conocimiento, pero esta conducta no correlacionó con el liderazgo transformacional $(\mathrm{r}=0,39 ; \mathrm{p}=0,084)$. En los colaboradores, el liderazgo transformacional y el transaccional mostraron una relación significativa con compartir conocimiento ( $\mathrm{r}=0,29 ; \mathrm{p}<0,001 ; \mathrm{r}=0,26 ; \mathrm{p}=0,001$, respectivamente). Se concluyó que en los líderes, el liderazgo transaccional se relaciona con la conducta de compartir conocimiento, y las recompensas tienen un rol importante. No se confirmó la relación entre liderazgo transformacional y la conducta de compartir conocimiento, pero ésta si se da en los colaboradores. Se sugiere a la cultura organizacional como variable mediadora en la relación entre liderazgo transformacional y compartir conocimiento.
\end{abstract}

Palabras clave: liderazgo transformacional, liderazgo transaccional, compartir conocimiento, gestión del conocimiento

\section{RELATIONSHIP BETWEEN TRANSFORMATIONAL AND TRANSACTIONAL LEADERSHIP AND THE SHARING OF KNOWLEDGE IN TWO SERVICE ORGANIZATIONS}

\begin{abstract}
The purpose of this paper was to study the relationship between transformational and transactional leadership and the sharing of knowledge. A descriptive correlational design with regression analysis and a casual sampling method were used. The sample comprised 150 employees from two companies in the city of Bogotá, .D.C., Colombia, of which 14 were leaders and 136 were collaborators. Instruments used were the Multi-factor Leadership Questionnaire developed by Bass and Avolio (2000) and adapted by Vega \& Zavala (2004), and the Escala de Compartir Conocimiento [Sharing Knowledge Scale] developed by Castañeda (2010). Statistical analysis was performed with IBM-SPSS STATISTIC 20.0. Among leaders it was found that $43 \%$ of them are characterized by a low effectiveness leadership (low transformational and transactional) and $40 \%$ of them show a full range leadership (high transformational and transactional). Among collaborators, sharing of knowledge scored between 4.3 and 5.4 /6.0; sharing experiences and ideas stand out and to a lesser level, sharing documents and knowledge. Among leaders, transactional leadership significantly correlated $(\mathrm{r}=0.63 ; \mathrm{p}=0.01)$ with sharing of knowledge but this behavior did not correlate with the transformational style $(\mathrm{r}=0.39 ; \mathrm{p}=0.084)$; while among collaborators, transformational and transactional
\end{abstract}

* Magister en Psicología, énfasis en Psicología Organizacional y del Trabajo, Universidad Católica de Colombia. Docente investigadora Universidad Santo Tomás, Bogotá Colombia. Edificio Dr. Angélico, Carrera 9 No. 72-90 Teléfono +57 4724358. Docente Universidad Santo Tomás. Bogotá, Colombia. magiduc15@hotmail.com

** PhD. Director de la Maestría en Psicología con énfasis en Psicología Organizacional y del Trabajo de la Universidad Católica de Colombia.dicastaneda@ucatolica.edu.co 
leadership showed a significant relationship with sharing of knowledge $(r=0.29 ; p<0.001 ; r=0.26 ; p=0.001$, respectively). It was concluded that from the perspective of the leaders, there is a relationship between transactional leadership and the behavior of sharing knowledge, and that rewards play an important role. Among leaders, the relationship between transformational leadership and sharing of knowledge was not confirmed, but it was confirmed in collaborators. Organizational culture is suggested as a mediating variable in the relationship between transformational leadership and the sharing of knowledge.

Key words: transformational leadership, transactional leadership, sharing of knowledge, knowledge management

\title{
RELAÇÃO ENTRE LIDERANÇA TRANSFORMACIONAL E TRANSACIONAL COM A CONDUTA DE COMPARTILHAR CONHECIMENTOS EM DUAS EMPRESAS DE SERVIÇOS
}

\author{
Resumo
}

\begin{abstract}
O objetivo foi descrever a relação entre a liderança transformacional-transacional do líder com a conduta de compartilhar conhecimento dos colaboradores. Utilizando um desenho descritivo-correlacional com análise regressional e uma amostragem incidental, avaliaram-se 150 trabalhadores de duas empresas de Bogotá: 14 líderes e 136 colaboradores. Os instrumentos aplicados foram o Multifactor Leadership Questionarie de Bass \& Avolio adaptado por Vega \& Zavala (2004) e a escala de Compartilhar Conhecimento de Castañeda (2010). A análise estatística realizou-se com o pacote estatístico IBM-SPSS STATISTIC 20,0. Entre os líderes encontrou-se que $43 \%$ se caracteriza por uma liderança de baixa efetividade (baixo nível transformacional e transacional) e $40 \%$, por uma liderança de nível completo (alto nível transformacional e transacional). Nos colaboradores, o compartilhar conhecimento pontuou entre 4,3 e 5,4/6,0; sobressaí compartilhar experiências e ideias e, em menor nível, compartilhar documentos e conhecimentos. Desde a perspectiva dos chefes, a liderança transacional correlacionou significativamente $(\mathrm{r}=0,63 ; \mathrm{p}=0,01)$ com compartilhar conhecimento, mas esta conduta não se correlacionou com a liderança transformacional $(\mathrm{r}=0,39 ; \mathrm{p}=0,084)$. Nos colaboradores, a liderança transformacional e a transacional mostraram uma relação significativa com compartilhar conhecimento $(\mathrm{r}=0,29 ; \mathrm{p}<0,001 ; \mathrm{r}=0,26 ; \mathrm{p}=0,001$, respectivamente). Conclui-se que nos líderes, a liderança transacional se relaciona com a conduta de compartilhar conhecimento, e as recompensas têm um papel importante. Não confirmou-se a relação entre liderança transformacional e a conduta de compartilhar conhecimento, mas a mesma se dá nos colaboradores. Sugere-se a cultura organizacional como variável mediadora na relação entre liderança transformacional e compartilhar conhecimento.

Palavras chave: liderança transformacional, liderança transacional, compartilhar conhecimento, gestão do conhecimento
\end{abstract}

\section{INTRODUCCIÓN}

En el contexto organizacional una de las conductas más esperadas en los directivos es el liderazgo, que no es una dimensión simple del comportamiento humano. Actualmente se diferencia entre liderazgo transformacional y liderazgo transaccional, que por su parte, parecen tener relación con la conducta de compartir conocimiento en el ámbito de la organización.

La relación entre el liderazgo transformacional-transaccional y la conducta de compartir conocimiento ha sido investigada bajo perspectivas predominantemente conceptuales (Maier \& Smith, 2007; Lu, Leung \& Tremain, 2006; Sun \& Hao, 2006; Avolio, Bass \& Jung, 1999); no obstante, en el entorno colombiano se carece de evidencia al respecto.

\section{La gestión del conocimiento}

La gestión del conocimiento ha cobrado interés dentro de las organizaciones debido a que el conocimiento es un recurso que aporta al fortalecimiento de las competencias diferenciadoras de una empresa (Salazar del Castillo, 2004). La gestión del conocimiento se define como la creación, organización, intercambio y uso de conocimiento con el fin de generar o mejorar productos y servicios, y de esta forma contribuir al logro de objetivos organizacionales (Castañeda, 2010). Un proceso adicional de este constructo es la protección del conocimiento (Karkoulian \& Masheredjian, 2012). La gestión del conocimiento requiere incentivar buenas prácticas que apoyen el flujo de éste (Maier \& Schmidt, 2007; Palacios \& Garrigós, 2006), así como un liderazgo activo, especialmente en la integración, asimilación (Avolio, Zhu, Koh \& Bhatia, 2004; Lakshman, 2005) y aplicación del mismo (Singh, 2008).

Uno de los aspectos esenciales para el éxito de iniciativas de gestión del conocimiento es la interacción social de los colaboradores debido a que son ellos quienes lo poseen (Nonaka \& Takeuchi, 1995; Smith, 2009). Esto es posible mediante la conducta de compartir conocimiento, la cual se entiende como un proceso dinámico de diálogo e 
intercambio de información entre personas en un contexto organizacional (Chennamaneni, 2006). De acuerdo con Shafieiyoun \& Safaei (2013), compartir conocimiento es considerado hoy el proceso más importante de la gestión del mismo, dado que facilita tanto su captura como aplicación (Christensen, 2007; Cummings, 2003). En las organizaciones, las personas comparten experiencias, experticia, información contextual, documentos e informes relacionados con el trabajo (Castañeda, 2010); sin embargo, hacerlo no es un proceso automático, sino que depende de variables como qué tanto la organización valora su conocimiento (Ford \& Staples, 2006), de si el trato es digno y las interacciones son recíprocas (Chennamaneni, 2006), tópicos que están relacionados con el tema de liderazgo.

\section{El liderazgo en el contexto organizacional}

El liderazgo es un proceso social de influencia, ya sea mediante la comunicación persuasiva o a través de la conducta (Sadler, 2003). En las organizaciones el liderazgo involucra una interacción entre dos partes: los líderes y los seguidores, quienes establecen una relación de doble vía, en pro de resultados (Eppard, 2004). El liderazgo influye en el proceso de trabajo en equipo, en el clima social, en la productividad individual y colectiva (Rodríguez-Ponce, 2007), en la calidad de vida de quienes lo ejercen (Juárez y Contreras, 2012), en indicadores de resultados como, por ejemplo, la satisfacción de los clientes (Belinska-Reformat \& Sztandret, 2013) y en la competitividad de la empresa (Bryant, 2003).

En la amplia gama de teorías actuales de liderazgo, la teoría de liderazgo transformacional-transaccional ha sido considerada de relevancia. El liderazgo transformacional tiene sus orígenes en las ideas de liderazgo carismático planteadas inicialmente por House (citado por Bass \& Riggio, 2006). Burns (citado por Bass, 1999) considera el liderazgo transformacional-transaccional como un solo constructo. Sin embargo, estudios empíricos han evidenciado que cada uno presenta dimensiones diferentes. Avolio, et al. (1999), creadores del modelo de liderazgo de rango completo o full range leadership model, destacan que los mejores líderes evidencian características de ambos estilos de liderazgo.

El liderazgo transformacional se define como un proceso social que se establece entre el líder y sus seguidores. El líder transformacional se caracteriza por ser carismático y por orientarse a conocer ampliamente a cada uno de los miembros de su equipo, de manera que les proporciona retos y oportunidades de desarrollo (Avolio, Eden \& Shamir, 2002; Bass, 1998; Bass, 1999). El liderazgo transformacional se compone de los siguientes sub-factores: el primero es carisma inspiracional, caracterizado por articular el futuro con una visión atractiva y una conducta ética, lo cual promueve la motivación y el desafío de sus seguidores. En el segundo, la influencia idealizada conductual, los líderes son percibidos por su equipo con respeto, debido a su alto grado de moralidad, carisma, confianza e integridad, actitud de escucha y de retroalimentación. En el tercero, influencia idealizada atribuida, los líderes logran confianza y respeto por parte de sus seguidores al ser un modelo conductual que facilita la identificación por parte del equipo. El cuarto, motivación inspiracional, se caracteriza porque los líderes llevan a cabo acciones a fin de que su equipo actúe de manera empoderada en el proceso de logro de metas articuladas a la visión organizacional, lo cual promueve su motivación. En el quinto, estimulación intelectual, los líderes desarrollan acciones que faciliten en sus seguidores oportunidades de desarrollo mediante el razonamiento sobre problemas organizacionales relevantes. Los líderes transformacionales promueven el aprendizaje organizacional conduciendo a sus colaboradores a descubrir modelos mentales para desarrollar una visión sistemática de la organización (García-Morales Llórens-Montes \& Verdú-Jover, 2008), así como el sentido de pertenencia y el compromiso, los cuales contribuyen a que se presente la conducta de compartir conocimiento (Carmeli, Atwater \& Levi, 2011).

El liderazgo transaccional se define como una relación entre el líder y sus seguidores que se fundamenta en el intercambio de recompensas asociadas a unas acciones. El liderazgo transaccional opera con base en autoridad burocrática y legitimidad dentro de la organización. Los líderes transaccionales enfatizan las tareas en los estándares de trabajo, así como en el logro de metas. Cuando el ambiente y el trabajo no favorecen la motivación y satisfacción del equipo, el uso de recompensas se constituye en un mecanismo eficaz para subsanar dichas carencias (Burns, citado por Mendoza, 2007). Bass y Avolio (2000) indican que los factores del liderazgo transaccional son: Primero, la consideración individualizada, caracterizada por la identificación de las necesidades de los miembros de su equipo a fin de facilitar su satisfacción. Segundo, la recompensa contingente, asociada al estilo transaccional clásico, que consiste en incentivar a los seguidores al hacer un reconocimiento de los logros obtenidos. Tercero, el liderazgo correctivo evitador, caracterizado por la identificación que hace el líder de las falencias que se presentan en el desempeño de sus seguidores y la toma de medidas correctivas. Cuarto, la dirección por excepción activa, consistente en que el líder monitorea e identifica desviaciones y errores con respecto a las normas e implementa acciones que conducen a su solución. Quinto, el liderazgo pasivo evitador, en el cual el líder implementa medidas correctivas únicamente 
después de que se ha presentado un problema mayor o no realiza ninguna acción. En el sexto, la dirección por excepción pasiva, la posición adoptada por el líder es actuar cuando surgen los problemas; es decir, únicamente interviene cuando las circunstancias lo ameritan debido a la gravedad del problema. Finalmente, en el laissez-faire, el líder evita tomar decisiones, no asume responsabilidad, ni usa la autoridad.

\section{Relación entre liderazgo transformacional-transaccional} y el compartir conocimiento

Existen estudios sobre la relación entre el liderazgo transformacional-transaccional y la conducta de compartir conocimiento con algunas diferencias en los resultados, las cuales son atribuidas principalmente a la cultura y sus componentes; sin embargo, ninguno de estos estudios ha sido realizado en el contexto colombiano y sólo uno en Latinoamérica, lo cual evidencia la necesidad de investigaciones en el área.

En el trabajo realizado por Delgado, Pedraja-Rejas \& Rodríguez-Ponce (2010) con pequeñas y medianas empresas en el norte de Chile, se halló que en la fase de crear conocimiento el estilo transformacional influye significativa y positivamente en la generación de nuevas ideas y su socialización. El liderazgo transaccional es irrelevante en esta fase. En la fase de compartir conocimiento, tanto el liderazgo transaccional como el transformacional son relevantes. En la fase de aplicar conocimiento ambos estilos de liderazgo son importantes; sin embargo, el liderazgo transaccional cobra mayor significancia. Los mejores líderes en estos dos procesos han mostrado un balance entre liderazgo transformacional y transaccional (Vega \& Zavala, 2004).

Chen \& Barnes (2006), en un estudio en organizaciones comercializadoras de servicios profesionales en Taiwán y Estados Unidos, encontraron que las acciones que caracterizan el estilo de liderazgo transformacional promueven la conducta de compartir conocimiento de sus seguidores. En cuanto al estilo de liderazgo transaccional, la recompensa contingente es un predictor de la conducta de compartir conocimiento. Yulk (2009) halló evidencia para afirmar que el líder transformacional tiende a promover la innovación en productos, servicios, procesos o tecnología, mientras que el líder transaccional enfatiza en el mejoramiento de los productos, servicios y procesos existentes.

Pauliene (2012) llama la atención sobre la importancia de tener en cuenta la variable cultura en la aplicación de modelos y teorías usados en contextos diferentes al de origen. Específicamente, proponen que el concepto de liderazgo puede variar según la cultura. En esa dirección, en el contexto japonés, considerado un país con una cultura colectivista, Perrin, et al. (2012) encontraron relación entre liderazgo transformacional y compartir conocimiento. Shao, Feng \& Liu (2012) en China hallaron que el liderazgo transformacional tiene una relación directa con la cultura organizacional e indirecto con la conducta de compartir conocimiento.

En el contexto coreano, Song, Kolb, Lee \& Kim (2012) hallaron que el liderazgo transformacional tiene relación con la creación de conocimiento y que el compromiso media la relación entre las dos variables. En la misma dirección existen autores que afirman que el liderazgo transformacional promueve un clima que incrementa la intención de compartir conocimiento (Liu \& DeFrank, 2013) y la conducta de compartir conocimiento (Shih, Chiang \& Chen, 2012). Carmeli, et al. (2011), señalan en su estudio en empresas de Israel, que los cargos de alto nivel tienden a no compartir con facilidad su conocimiento en el sitio de trabajo, lo cual se explica parcialmente por temor a perder control. El estudio encontró que la identificación relacional de los colaboradores está mediada por la relación líder-miembro, lo cual contribuye a un mayor sentido de pertenencia con la organización, así como a facilitar la conducta de compartir conocimiento.

Con base en la conceptualización y la evidencia empírica, el objetivo de este estudio fue indagar sobre la relación entre el liderazgo transformacional y transaccional de los líderes con la conducta de compartir conocimiento de los colaboradores, empleados de dos organizaciones empresariales, una entidad de servicios de software y la sección de bibliotecas públicas de una caja de compensación familiar. A partir de ello se pretendió ajustar un modelo predictivo de la conducta de compartir conocimiento de los colaboradores en función de los niveles de liderazgo transformacional y transaccional de los líderes.

Como hipótesis se planteó que tanto el estilo de liderazgo transformacional (H1) como el liderazgo transaccional (H2) de los directivos se relacionan directa y significativamente con la conducta de compartir conocimiento de los colaboradores y, por ende, se pueden establecer modelos predictivos significativos (H3) de la conducta de compartir conocimiento en función de los niveles de liderazgo transformacional y transaccional.

\section{MÉTODO}

\section{Diseño}

Se utilizó un diseño multivariable descriptivo-correlacional, con análisis regresional (Hernández, Fernández \& Baptista, 2006). Este diseño permitió caracterizar el comportamiento de las variables e identificar las relaciones 
que no son evidentes con la pura descripción univariable. Además, se asume que en el ámbito de la covariación de variables, y sin realizar manipulaciones experimentales ni hacer explicaciones causales, es metodológicamente válido desarrollar modelamientos predictivos, buscando representar funcionalmente la relación multivariable que se encuentre (Hair, Anderson, Tatham \& Black, 2000).

\section{Participantes}

En el estudio participaron ciento cincuenta trabajadores vinculados a dos organizaciones ubicadas en la ciudad de Bogotá, Colombia. La primera, una empresa prestadora de servicios de software, en la cual ocho personas tenían el rol de líderes y 97 de colaboradores; la prueba se aplicó a directivos y coordinadores administrativos y a sus colaboradores. La segunda fue la sección de bibliotecas públicas de una caja de compensación familiar en la cual seis personas tenían el rol de líderes y 39 de colaboradores. El instrumento se aplicó a directores de bibliotecas y a miembros de sus equipos de trabajo. La participación fue voluntaria, previa claridad del consentimiento informado.

Considerando teóricamente que no había diferencias en las funciones misionales de prestación de servicios de las dos empresas que generasen efectos en las variables de medida (Castañeda, 2010), las dos entidades se eligieron estrictamente por su accesibilidad y manifiesta colaboración con la investigación en términos de la recolección de la muestra. Se tomó la totalidad de los empleados sin hacer selección aleatoria o intencional y la conformación y diseño muestral se configuró como un modelo de muestreo no probabilístico de tipo incidental por disponibilidad (Daniel, 2002), tanto de las empresas colaboradoras como de los participantes.

\section{Variables}

Se definieron como variables predictoras el liderazgo transformacional y el liderazgo transaccional de los directivos, En la variable liderazgo transformacional están involucrados los factores de: carisma inspiracional, influencia idealizada conductual, influencia idealizada atribuida, motivación inspiracional y estimulación intelectual; mientras que en el liderazgo transaccional los factores son: consideración individualizada, recompensa contingente, liderazgo correctivo evitador, dirección por excepción activa, liderazgo pasivo-evitador, dirección por excepción pasiva y laissez-faire.

Como variable criterio se definió a la conducta de compartir conocimiento de los subalternos, que incluye los factores de compartir conocimientos, compartir experiencias, compartir ideas y compartir documentos.

\section{Instrumentos}

Para el estilo de liderazgo se utilizó el Multifactor Leadership Questionnaire (MLQ) de Bass \& Avolio (2000), que mediante una escala tipo Likert mide tanto el liderazgo transformacional como el transaccional, al igual que los factores particulares que los componen, mencionados en la definición de variables y definidos en la revisión teórica previa. Este instrumento fue diseñado por sus autores de tal manera que permite valorar el liderazgo según las respuestas de forma independiente de los líderes y de los subalternos, para su posible comparación o correlación, según desee el investigador o se establezca en los objetivos del estudio.

En lo que a su rango de variación se refiere, la forma de calificación de la escala y subescalas de la prueba se ciñen al dominio numérico valorativo dado por los ítems (0-4), dentro del cual el promedio individual o grupal de escalas y subescalas se interpreta de tal manera que un mayor puntaje indica un mayor grado de liderazgo transformacional o transaccional y viceversa, controlándose con ello también el efecto del número de ítems de las subescala para efecto de su comparabilidad. Se utilizó la adaptación castellana en contexto latinoamericano realizada por Vega \& Zavala (2004), que reportaron que esta versión tiene un nivel de confiabilidad de 0,97 .

Por otra parte, para la conducta de compartir conocimiento se utilizó la escala de igual nombre del instrumento Variables Psicosociales y Condiciones Organizacionales de la Conducta de Compartir Conocimiento, diseñada y validada por Castañeda (2010) con población colombiana con base en el modelo logístico de Rasch (Prieto \& Delgado, 2003). Es una escala tipo Likert de siete niveles de respuesta para valorar los factores respectivos, que ya se han mencionado. Su rango de variación también se refiere a la forma de calificación de la escala y subescalas de la prueba y se ciñen al dominio numérico valorativo dado por los ítems (0-6), dentro del cual un promedio, sea individual o grupal, se interpreta de tal manera que a mayor puntaje, mayor ocurrencia de la conducta de compartir conocimiento y viceversa. El nivel de confiabilidad reportado con el coeficiente Alpha de Cronbach fue 0,81, el cual se considera "adecuado", en términos de consistencia interna (Campo-Arias \& Oviedo, 2008).

\section{Procedimiento}

Con el apoyo de directivos de las dos entidades se convocó a todos los empleados a participar voluntariamente en el estudio. La aplicación de los instrumentos se hizo en forma presencial en los puestos de trabajo de los participantes, previa aceptación del consentimiento informado y explicación sobre los objetivos del estudio. 
Con el propósito de identificar los comportamientos de los líderes y poderlos relacionar con la conducta de compartir conocimiento de los colaboradores, se obtuvo tanto la autovaloración de los líderes como la valoración que de sus seguidores en los formatos paralelos del MLQ adaptados por Vega \& Zavala (2004) y la escala Compartir conocimiento de Castañeda (2010).

La aplicación se realizó durante momentos de baja intensidad de las actividades laborales para evitar interferencias e interrupciones; igualmente se dieron las orientaciones necesarias para diligenciar adecuadamente los formatos, sin llegar a incidir sobre las respuestas y al final se verificó que todos los ítems fueran respondidos a cabalidad.

\section{Plan de análisis}

El análisis de datos se realizó con el software IBM SPSS STATISTIC versión 20,0. Inicialmente se realizó el análisis descriptivo. Se obtuvieron los estadísticos de tendencia central y dispersión para las variables numéricas, mientras que para las variables categóricas se elaboró la distribución porcentual de frecuencias.

Para el análisis correlacional se calcularon los coeficientes bivariados producto-momento de Pearson (Daniel, 2002) entre los puntaje de liderazgo transformacional y transaccional con la conducta de compartir conocimiento, de manera que los dos grupos de las dos entidades dieron respuesta a los dos instrumentos: Liderazgo y Compartir conocimiento, lo cual permite la correlación sin llegar a mezclar las muestras que, per sé, no están balanceadas.

A partir del trasfondo correlacional, se realizaron modelamientos multivariables con la técnica de regresión lineal múltiple y la opción step waise forward (Hair, et al. 2000). Ésta valora inicialmente la significancia predictiva de todos los variables, y el modelo selecciona las predictoras con mayor aporte significativo a la estimación de una variable criterio, no incluyendo en la ecuación final aquellos predictores que no superan el nivel de significancia, con lo cual se obtuvo una ecuación de regresión ajustada, que bajo condiciones muestrales similares, al desplegarla permitiría la estimación de la conducta de compartir conocimiento de los colaboradores según los niveles de liderazgo de los directivos organizacionales.

En el análisis de datos se definió como criterio de significancia una $\mathrm{p} \leq 0.05$ para la aceptación/rechazo de las hipótesis estadísticas subyacentes (Daniel, 2002), así como también para la inclusión/eliminación de predictores en el ajuste multivariable de las ecuaciones de regresión.

\section{RESULTADOS}

Caracterización del estilo de liderazgo y el compartir conocimiento

Con base en las respuestas al MLQ de los 14 líderes y sus 136 seguidores en las dos empresas participantes, se identificó la tipología de los estilos de liderazgo transformacional y transaccional (véase Figura 1).

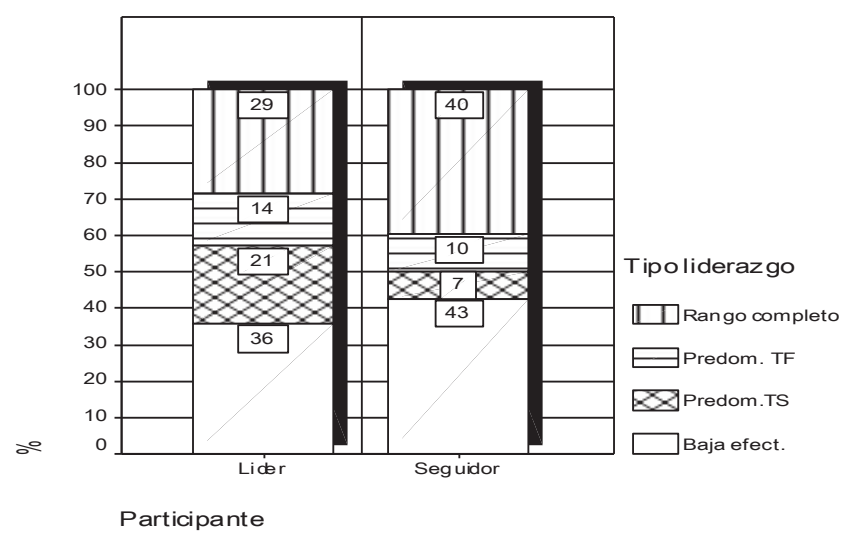

Figura 1. Tipología de liderazgo transformacional y transaccional de los líderes.

En la muestra de líderes, a partir de su autodescripción y aplicando la categorización propuesta por Avolio, Bass y Jung (1999), se identificó que el 36\% presenta un liderazgo de "baja efectividad", es decir, tienen bajo liderazgo transformacional y bajo liderazgo transaccional, mientras que el $29 \%$ presenta un liderazgo de rango completo (alto liderazgo transformacional y alto liderazgo transaccional). El 21\% corresponde a líderes predominantemente transaccionales y el $14 \%$ a líderes predominantemente transformacionales.

Por su parte, en la valoración que hacen los colaboradores de sus líderes, $43 \%$ presenta un liderazgo de baja efectividad (bajo liderazgo transformacional y bajo liderazgo transaccional), $40 \%$ un liderazgo de rango completo (alto liderazgo transformacional y alto liderazgo transaccional), $7 \%$, liderazgo predominantemente transaccional y $10 \%$ liderazgo predominantemente transformacional. Al analizar las dos distribuciones, las diferencias proporcionales no fueron significativas ( $\mathrm{p}=0,303$ ), lo cual sugiere que los estilos de liderazgo son percibidos de manera similar entre los líderes y sus colaboradores y es llamativo que en ambas valoraciones sobresale el liderazgo de "baja efectividad", que corresponde tanto a un bajo nivel de liderazgo transformacional como de liderazgo transaccional, aun cuando 
seguidamente aparece en proporción el estilo de "rango completo" que implica la presencia de un alto nivel de liderazgo transformacional y también transaccional.

En lo que se refiere a la conducta de compartir conocimiento, la figura 2 muestra el perfil de percepción de los líderes, así como también la valoración según sus seguidores.

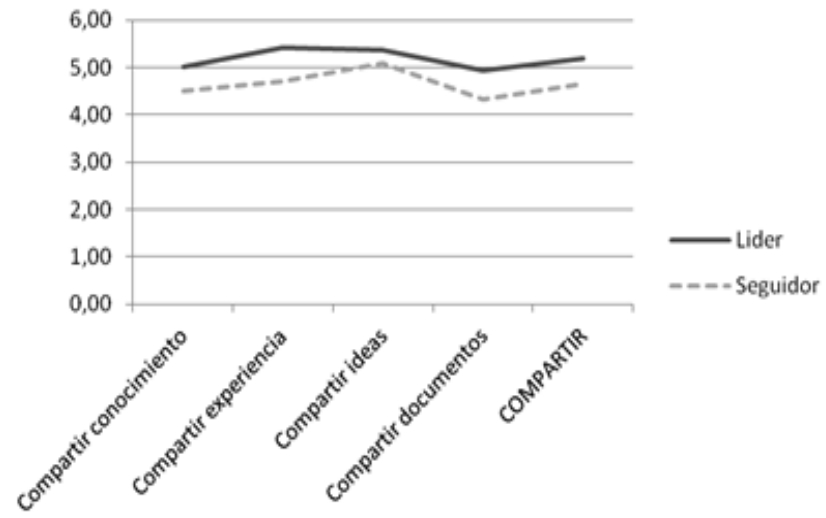

Figura 2. Perfil comparativo de la conducta de compartir conocimiento según los líderes y los seguidores.

Se aprecia que los promedios en las dimensiones de compartir conocimiento de los empleados oscilan entre 4,3 y 5,4 sobre un rango de 6,0 , que son valores relativamente altos en las conductas de compartir conocimiento, ya sea en términos de experiencias, ideas o documentos.

Adicionalmente, según los puntajes de los líderes, sobresale el compartir experiencias e ideas, y en menor grado, el compartir documentos. Mientras que en la valoración reali- zada por los seguidores se destaca que se comparten ideas en mayor grado, y en menor grado se comparten documentos, pero de todos modos, se hace notar que la percepción de la conducta de compartir conocimiento es mayor por parte de los líderes que de los seguidores, en todas las dimensiones de expresión de esta conducta.

\section{Análisis correlacional del estilo de liderazgo y el com- partir conocimiento}

Pasando al análisis relacional entre el estilo de liderazgo del directivo con la conducta de compartir conocimiento de los seguidores, en la tabla 1 se presenta la matriz de correlaciones resultante, diferenciando entre la valoración realizada por líderes y subalternos.

En el ámbito de la evaluación realizada por los líderes, el nivel de liderazgo transaccional de los directivos presentó una correlación directa media significativa $(\mathrm{r}=0,629$; $\mathrm{p}=0,01$ ) con la conducta de compartir conocimiento de los subalternos, mientras que el liderazgo transformacional no correlacionó significativamente con dicha conducta $(\mathrm{r}=0,39$; $\mathrm{p}=0,084)$. En conjunto, tales datos permiten afirmar que los líderes que tienen mayor nivel de liderazgo transaccional sus seguidores presentan igualmente un mayor nivel en la conducta de compartir conocimiento, tendencia que no se observa con el liderazgo transformacional.

Con base en ello, desde la perspectiva de los líderes no se acepta la hipótesis que propone que el estilo transformacional presenta correlación directa con la conducta de compartir conocimiento, mientras que sí se acepta la

Tabla 1.

Matriz de correlaciones del liderazgo y la conducta de compartir conocimiento

\begin{tabular}{|c|c|c|c|c|c|}
\hline \multicolumn{6}{|c|}{ Correlaciones } \\
\hline \multicolumn{2}{|r|}{ Participante } & & LDZGO. & LDZGO. & COMPARTIR \\
\hline \multirow{6}{*}{ Líder } & \multirow{2}{*}{ LDZGO. TRANSFORMACIONAL } & $\mathrm{r}$ & 1 &, $759 * *$ & 390 \\
\hline & & Sig. & &, 001 & 084 \\
\hline & \multirow{2}{*}{ LDZGO. TRANSACCIONAL } & $\mathrm{r}$ &, $759 * *$ & 1 &, $629 * *$ \\
\hline & & Sig. &, 001 & &, 008 \\
\hline & \multirow{2}{*}{ COMPARTIR } & $\mathrm{r}$ & ,390 &, $629 * *$ & 1 \\
\hline & & Sig. &, 084 &, 008 & \\
\hline \multirow{6}{*}{ Seguidor } & \multirow{2}{*}{ LDZGO. TRANSFORMACIONAL } & $\mathrm{r}$ & 1 &, $893 * *$ &, $292 * *$ \\
\hline & & Sig. & &, 000 &, 000 \\
\hline & \multirow{2}{*}{ LDZGO. TRANSACCIONAL } & $\mathrm{r}$ &, $893 * *$ & 1 &, $264 * *$ \\
\hline & & Sig. &, 000 & &, 001 \\
\hline & \multirow{2}{*}{ COMPARTIR } & $\mathrm{r}$ &, $292 * *$ &, $264 * *$ & 1 \\
\hline & & Sig. &, 000 &, 001 & \\
\hline
\end{tabular}

** La correlación es significante al nivel 0,01 
segunda hipótesis que sugiere que el estilo de liderazgo transaccional se relaciona positivamente con la conducta de compartir conocimiento.

Por otra parte, pasando ahora a la evaluación realizada a los seguidores, tanto el liderazgo transformacional como el transaccional del líder presentaron una relación directa significativa, aunque baja, con la conducta de compartir conocimiento ( $\mathrm{p}<0,001$ y $\mathrm{p}=0,001$, respectivamente) autovalorada por los seguidores; de manera que se puede afirmar que los subalternos que califican a sus líderes con alto nivel de liderazgo transformacional y transaccional presentan un puntaje mayor en la conducta de compartir conocimiento.

De forma complementaria, también se realizó un análisis correlacional de los estilos de liderazgo con las dimensiones de la conducta de compartir conocimiento (véase Tabla 2).

En la autodescripción de los líderes se encontró que el liderazgo transformacional y el transaccional del líder correlacionan de manera directa significativa solo con la conducta de los seguidores en compartir ideas $(\mathrm{r}=0,595 \mathrm{y}$ $\mathrm{r}=0,768$, respectivamente) y no con el compartir experiencia, conocimiento, documentos. Así, se puede afirmar que tanto un alto estilo transformacional como un alto estilo transaccional de los líderes se relacionan con una mayor expresión de la conducta de compartir ideas de los subalternos, aun cuando esto mismo no ocurre con las conductas de compartir experiencias, conocimientos o documentos.

De otra parte, en la valoración hecha por los seguidores sobre el liderazgo de sus jefes y de su propia conducta de compartir conocimiento, se encontró que tanto el nivel de liderazgo transformacional como el transaccional del líder correlacionan directa y significativamente con compartir ideas, experiencias y documentos; de manera que, según los subalternos, un alto estilo transformacional y transaccional de los directivos está relacionado con mayor expresión comunicativa de ideas, experiencias e intercambio de do- cumentos y por ende, también un bajo nivel de liderazgo transformacional y transaccional del directivo se relaciona con un menor nivel de compartir conocimientos en términos de ideas, experiencias y documentos.

Así mismo, al realizar el análisis correlacional de los factores del estilo de liderazgo con la conducta de compartir conocimiento (véase Tabla 3), según la autodescripción de los lideres, se puede afirmar que los niveles altos de estimulación inspiracional, consideración individualizada, recompensa contingente, dirección por excepción activa y pasiva, laiseez-faire y liderazgo pasivo-evitador, se relaciona significativamente con una mayor expresión de la conducta de compartir conocimiento de los subalternos.

Por su parte, en el ámbito de la valoración de los seguidores, se encontró que casi todos los factores del estilo de liderazgo del jefe se relacionan significativamente con la conducta de compartir conocimiento de los subalternos, excepto la Dirección por excepción activa y pasiva, el Laiseez-faire y el Liderazgo pasivo-evitador.

\section{Análisis predictivo del compartir conocimiento según el liderazgo transformacional-transaccional}

Finalmente, se realizó un análisis de regresión múltiple que permitiese el modelamiento predictivo de la conducta de compartir conocimiento por parte de los seguidores, en función de los niveles de liderazgo transformacional y transaccional del líder (véase Tabla 4). Se utilizó la técnica step waise forward que selecciona progresivamente las variables relacionadas de mayor significancia para conformar el modelo de mejor ajuste predictivo (Hair, et al. 2000), eliminando las variables predictoras que no hacen un aporte significativo a la estimación global de la variable criterio. Se hace notar que debido a las diferencias en el tamaño de las muestras de líderes y subalternos se hicieron análisis

Tabla 2.

Matriz de correlaciones del liderazgo con las dimensiones de compartir conocimiento.

\begin{tabular}{|c|c|c|c|c|c|c|}
\hline \multicolumn{7}{|c|}{ Correlaciones } \\
\hline & Participante & & $\begin{array}{c}\text { Compartir } \\
\text { conocimiento }\end{array}$ & $\begin{array}{c}\text { Compartir } \\
\text { experiencia }\end{array}$ & $\begin{array}{c}\text { Compartir } \\
\text { ideas }\end{array}$ & $\begin{array}{l}\text { Compartir } \\
\text { documentos }\end{array}$ \\
\hline \multirow{4}{*}{ Líder } & \multirow{2}{*}{ LDZGO. TRANSFORMACIONAL } & $\mathrm{r}$ & ,299 &, 060 &, 595 &, 110 \\
\hline & & Sig. & ,149 & ,420 &, 012 & ,354 \\
\hline & \multirow{2}{*}{ LDZGO. TRANSACCIONAL } & $\mathrm{r}$ & ,366 & ,286 & ,768 & ,314 \\
\hline & & Sig. & ,099 & , 161 &, 001 & , 137 \\
\hline \multirow{4}{*}{ Seguidor } & \multirow{2}{*}{ LDZGO. TRANSFORMACIONAL } & $\mathrm{r}$ &, 140 & ,279 & ,262 & ,266 \\
\hline & & Sig. &, 052 &, 000 &, 001 & ,001 \\
\hline & \multirow{2}{*}{ LDZGO. TRANSACCIONAL } & $\mathrm{r}$ &, 115 & ,230 & ,225 & ,276 \\
\hline & & &, 091 &, 004 &, 004 &, 001 \\
\hline
\end{tabular}


de regresión independientes en cada grupo, con lo cual se hace la estimación sin mezclar las muestras, que no están balanceadas.

En el ámbito de la autovaloración del líder, el liderazgo transaccional se muestra como el predictor significativo $(\mathrm{p}=0,016)$ de la conducta de compartir conocimiento de los seguidores, mientras que el liderazgo transformacional no se mostró como estimador relevante $(\mathrm{p}>0,05)$ y no logró aportar a la predicción del compartir conocimiento, por lo cual no fue incluido en el modelo funcional, que para el efecto arrojó la ecuación de estimación ajustada Ec.1.

$$
\hat{C}=2,72+0,73 L T S \quad \text { Ec. } 1
$$

Donde: Ĉ: Puntaje estimado Conducta de compartir conocimiento del subalterno

LTs: Puntaje observado de Liderazgo Transaccional del directivo

De esta manera, desde la perspectiva de los directivos, se puede interpretar que si el nivel de liderazgo transaccional del líder se incrementa, la expresión de la conducta de compartir conocimiento de los seguidores también se vería fortalecida y viceversa.

Por su parte, en contraposición, a partir de la valoración de los subalternos se pudo identificar que en este ámbito es el estilo de liderazgo transformacional del líder el que

Tabla 3.

Análisis relacional de los factores de liderazgo de los directivos con el compartir conocimiento de los subalternos: valoración realizada de manera independiente por líderes y por seguidores

\begin{tabular}{lllll}
\hline \multirow{2}{*}{ Subfactores } & \multicolumn{2}{c}{ Correlaciones } & \multicolumn{2}{c}{ SEGUIDORES } \\
\cline { 2 - 5 } & \multicolumn{2}{c}{ COMPARTIR } & \multicolumn{2}{c}{ COMPARTIR } \\
\cline { 2 - 5 } & $\mathrm{r}$ & Sig. & $\mathrm{r}$ & Sig. \\
\hline Influencia Ideal. Atribuida &, 118 &, 344 &, $247^{* *}$ &, 002 \\
\hline Influencia Ideal. Cdtual &, 121 &, 340 &, $214^{* *}$ &, 006 \\
\hline Carisma &, 141 &, 315 &, $243^{* *}$ &, 002 \\
\hline Motivación Inspiracional &, 388 &, 085 &, $288^{* *}$ &, 000 \\
\hline Carisma Inspiracional &, 256 &, 189 &, $268^{* *}$ &, 001 \\
\hline Estimulación Inspiracional &, $620^{* *}$ &, 009 &, $327^{* *}$ &, 000 \\
\hline Consider. Individualizada &, $500^{*}$ &, 034 &, $218^{* *}$ &, 005 \\
\hline Recompensa Contingente &, $635^{* *}$ &, 007 &, $290^{* *}$ &, 000 \\
\hline Dir. por Excepc. Activa &, $510^{*}$ &, 031 &, 121 &, 080 \\
\hline Dir. por Excepc. Pasiva &, $480^{*}$ &, 041 &,- 057 &, 256 \\
\hline Laiseez-Faire &, $595^{*}$ &, 012 &,- 019 &, 412 \\
\hline Lid. Pasivo-Evitador &, $559^{*}$ &, 019 &,- 045 &, 301 \\
\hline
\end{tabular}

* La correlación es significante al nivel 0,05

** La correlación es significante al nivel 0,01

Tabla 4.

Análisis de regresión múltiple step waise forward de la conducta de compartir conocimiento en función del nivel de liderazgo

\begin{tabular}{|c|c|c|c|c|c|c|}
\hline \multicolumn{7}{|c|}{ Coeficientes $^{\mathrm{a}}$} \\
\hline \multicolumn{7}{|c|}{ Coeficientes no estandarizados } \\
\hline \multicolumn{3}{|c|}{ Participante modelo } & $\mathrm{B}$ & Error típ. & $\mathrm{t}$ & Sig. \\
\hline \multirow{2}{*}{ Líder } & \multirow{2}{*}{1} & \multirow{2}{*}{$\begin{array}{l}\text { (Constante) } \\
\text { LDZGO. TRANSACCIONAL }\end{array}$} & 2,715 &, 886 & 3,063 & .010 \\
\hline & & & ,727 & ,260 & 2,800 & ,016 \\
\hline \multirow{2}{*}{ Seguidor } & \multirow{2}{*}{1} & (Constante) & 2,924 & ,499 & 5,858 & ,000 \\
\hline & & $\begin{array}{l}\text { LDZGO. } \\
\text { TRANSFORMACIONAL }\end{array}$ &, 563 &, 159 & 3,538 &, 001 \\
\hline
\end{tabular}

a. Variable dependiente: COMPARTIR 
se muestra como mejor predictor $(\mathrm{p}=0,001)$ de la conducta de compartir conocimiento, mientras que el liderazgo transaccional fue el que no logró ser predictor significativo $(p>0,05)$ al no aportar a la estimación del compartir conocimiento, lo que llevó a ser eliminado como predictor. El modelo de regresión ajustado arrojó la ecuación de predicción Ec. 2.

$$
\hat{C}=2,92+0,57 \operatorname{LTf} \quad \text { Ec. } 2
$$

\section{Donde: $\hat{\mathbf{C}}$ : Puntaje Estimado Conducta de Compartir conocimiento}

LTs: Puntaje Observado de Liderazgo Transformacional del Directivo

Así, desde la perspectiva de los subalternos, se podría afirmar que ante un mayor puntaje en el nivel de liderazgo transformacional del líder, la expresión de la conducta de compartir conocimiento de los seguidores igualmente se incrementaría, y viceversa.

\section{DISCUSIÓN}

El mundo organizacional actual está sometido a frecuentes exigencias y consideraciones de competencia donde el éxito es una demanda diaria que compromete todo el talento humano y es ahí donde se debe configurar el trabajo colaborativo bajo la guía y motivación de un líder que potencie la búsqueda de las metas corporativas y el crecimiento, tanto de la entidad, como de los individuos que la componen. Es así como durante los últimos tiempos, la gestión del conocimiento ha cobrado un alto interés dentro de las empresas, no sólo como parte fundamental dentro de las estrategias de crecimiento dentro del mercado, sino del direccionamiento de las mismas en general.

De acuerdo con los resultados de la investigación, tanto desde la perspectiva de los líderes como de los colaboradores, existe una relación entre liderazgo transaccional y la conducta de compartir conocimiento, dado que encontró apoyo estadístico para la hipótesis (H2) que así lo proponía. No ocurrió lo mismo con la otra hipótesis (H1) sobre la relación entre liderazgo transformacional y la conducta de compartir conocimiento, puesto que se halló respaldo estadístico para tal relación en las percepciones de los colaboradores pero no desde la perspectiva de los líderes. Adicionalmente, a partir de los datos no se hallaron diferencias significativas entre liderazgo transformacional y transaccional en relación con la conducta de compartir conocimiento, de manera que se da una relación similar entre el liderazgo transformacional y la conducta de compartir conocimiento, al igual que entre el liderazgo transaccional y dicha conducta.
De acuerdo con algunos autores, para las organizaciones es de gran importancia contar con líderes que desarrollen ambos estilos de liderazgo en un alto nivel, a los cuales se puede definir como líderes de rango completo (Avolio et al., 1999). Para otros autores es esencial que cada líder desarrolle la habilidad de distinguir el estilo apropiado según el contexto organizacional (Vega \& Zavala, 2004). En el presente estudio, de acuerdo con la auto-descripción de los líderes, sólo el 29\% registró puntuaciones altas en liderazgo transformacional y en liderazgo transaccional. Desde la perspectiva de los colaboradores el porcentaje de líderes de rango completo fue de $40 \%$.

Si lo anterior se analiza a la luz de los hallazgos de esta investigación, no es necesario ser líder de rango completo para influir en la conducta de compartir conocimiento, sino que dicha conducta está relacionada con el liderazgo transaccional desde las evaluaciones de líderes y colaboradores y con el liderazgo transformacional desde la perspectiva de colaboradores. Si el porcentaje de líderes en una organización que pueden ser descritos de rango completo es limitado, a partir de los datos se puede afirmar que esto no es una restricción para que se promueva el compartir conocimiento en las organizaciones. De esta forma, lo planteado por Avolio et al (1999) es deseable, mas no una limitación para compartir conocimiento.

El hallazgo de que para los participantes del estudio el liderazgo transaccional se relaciona con la conducta de compartir conocimiento, es consistente con lo encontrado por Delgado et al. (2010) y por Chen \& Barnes (2006). En el presente estudio el rol de las recompensas contingentes fue un factor relevante en la explicación de la relación entre liderazgo transaccional y la conducta de compartir conocimiento. Cuando la organización cuenta con incentivos para compartir conocimiento, y se utilizan desde un estilo de liderazgo transaccional, se incrementa la probabilidad de compartir conocimiento. Al respecto, Bartol \& Srivastava (2002) afirman que el conocimiento es un intangible que se comparte cuando hay razones económicas y no económicas para hacerlo. Las recompensas facilitan el proceso de socialización del conocimiento.

En contraposición a lo anterior, de acuerdo con la descripción de los seguidores, el nivel de liderazgo transformacional presentó relación con la conducta de compartir conocimiento, mientras que desde la perspectiva de los líderes esta relación no se encontró. Estudios previos como los de Delgado et al. (2010) y Chen \& Barnes (2006), apoyan la relación. Una posible explicación del porqué desde la perspectiva de líderes la correlación no fue significativa es sugerida por Yulk (2009), quien afirma que entre liderazgo transformacional y la conducta de compartir conocimiento interviene la cultura. Lo anterior es respaldado por Pauliene 
(2012), quien afirma que la relación entre liderazgo transformacional y la conducta de compartir conocimiento está mediada por la cultura organizacional. El rol de la cultura en la conducta de compartir conocimiento ha sido explorado por autores como McDermott \& Dell (2001) y el de la cultura en el liderazgo por Mendoza \& Ortiz (2006).

En futuras investigaciones se sugiere indagar si el rol de los participantes, es decir de líder o colaborador influye en las expectativas sobre compartir conocimiento. A la vez, el rol de la cultura en la relación entre tipo de liderazgo y la conducta de compartir conocimiento. Otra posible línea de investigación sobre el tipo de liderazgo y la conducta de compartir conocimiento es el nivel de desarrollo de las organizaciones. Es decir, es posible que en una organización de punta dedicada a la innovación, el liderazgo transformacional tenga un papel diferente sobre compartir conocimiento que en una organización proveedora de servicios. Al respecto, Liu \& DeFrank (2013) sugieren que el clima de confianza que promueven los líderes transformacionales se relaciona con compartir conocimiento. Adicionalmente, en organizaciones de avanzada la cultura organizacional está orientada al aprendizaje (Castañeda, 2010; Gammelgaard, Husted \& Michailova, 2004), los colaboradores conocen la estrategia organizacional y por lo tanto saben qué conocimiento es relevante de compartir (Liao, 2006; Zack, 2005), e igualmente se cuenta con los recursos tecnológicos y de apoyo para compartir dicho conocimiento (Lu, Leung \& Koch, 2006).

En este contexto también es necesario que en próximas investigaciones se explore el efecto que puedan tener las funciones misionales de las entidades, toda vez que si bien en este estudio se asumió a priori que era un factor no condicionante de las variables de medida, es posible que al explorar específicamente tal hipótesis se encuentre evidencia significativa, dado que la expresión del liderazgo posiblemente varíe en función de variables de cultura organizacional íntimamente ligadas a la misión y visión que en su planeación estratégica haya asumido la organización.

En conclusión, teniendo en cuenta que el propósito de esta investigación fue indagar sobre el rol del tipo de liderazgo sobre la conducta de compartir conocimiento, de acuerdo con los hallazgos de esta investigación, se puede afirmar con suficiente evidencia que el liderazgo transaccional efectivamente tiene relación con esta conducta. Los incentivos, característicos de este tipo de liderazgo, tienen un rol importante en la explicación. En relación con el liderazgo transformacional, en el cual la inspiración y el carisma del líder son más importantes que las recompensas para influir en las conductas de los colaboradores, en este aspecto los hallazgos no evidencian consenso entre líderes y colaboradores. Se sugiere ahondar en constructos como la cultura organizacional para entender de manera más profunda los hallazgos.

\section{REFERENCIAS}

Avolio, B., Bass, B., \& Jung, D. (1999). Re-examining the Components of Transformational and Transactional Leadership Using the Multifactor Leadership Questionnaire. Journal of Occupational Psychology. 72, 441-462.

Avolio, B., Eden, D., \& Shamir, B. (2002). Impact of Transformational Leadership on Follower Development and Performance: A Field Experiment. Academy of Management Journal. 45, 735-744.

Avolio, B., Zhu, W., Koh, W., \& Bathia, P. (2004). Transformational Leadership and Organizational Commitment: Mediating Role of Psychological Empowerment and Moderating Role of Structural Distance. Journal of Organizational Behavior. 25, 951-968.

Bartol, K., \& Srivastava, A. (2002). Encouraging Knowledge Sharing: The Role of Organizational Reward Systems. Journal of Leadership \& Organizational Studies. 9(1) 64-76.

Bass, B. (1998). Leading in the Army After Next. Military Review. 78(2), 46-57.

Bass, B. (1999). Two Decades of Research and Development in Transformational Leadership. European Journal of Work and Organizational Psychology. 8(1), 9-32.

Bass, B., \& Avolio, B. (2000). MLQ Multifactor Leadership Questionnaire. Second Edition. Redwood: Mind Garden Inc.

Bass, B., Einstein, W., \& Waldman, D. (1987). Leadership and Outcomes of Performance Appraisal Processes. Journal of Occupational Psychology. 60, 177-186. Bass, B., \& Riggio, R. (2006). Transformational Leadership. Second Edition. London: Lawrence Erlbaum Associates, Publishers.

Belinska-Reformat, K., \& Sztandret, I. (2013). Influence of Knowledge Sharing Between Intermediaries and IT Leaders on Developing Offers to Customers-Polish Perspective. International Journal of Management Cases. 15(4), 205-233.

Bryant, S. (2003). The Role of Transformational and Transactional Leadership in Creating, Sharing and Exploiting Organizational Knowledge. The Journal of Leadership and Organizational Studies. 9(4), 32-44.

Campo-Arias, Ay Oviedo, H. (2008). Propiedades psicométricas de una escala: La consistencia interna. Revista de Salud pública. 10(5): 831-839.

Carmeli, A., Atwater, L., \& Levi, A. (2011). How Leadership Enhances Employee's Knowledge Sharing: The Intervening Roles of Relational and Organizational Identification. Journal of Technology Transfer, 36(3), 257-274.

Castañeda, D. I. (2010). Variables psicosociales y condiciones organizacionales intervinientes en la intención y conducta de compartir conocimiento. (Tesis doctoral no publicada). Universidad Autónoma de Madrid, España. 
Chen, L.Y., \& Barnes, B. (2006). Leadership Behaviors and Knowledge Sharing in Professional Service Firms Engaged in Strategic Alliances. Journal of Applied Management and Entrepreneurship, 11(2), 51-69.

Chennamaneni, A. (2006). Determinants of Knowledge Sharing Behaviors: Developing and Testing an Integrated Theoretical Model. (Unpublished doctoral dissertation). The University of Texas at Arlington.

Christensen, P. (2007). Knowledge Sharing: Moving Away from the Obsession with Best Practices. Journal of Knowledge Management, 11(1) 36-47.

Cummings J. (2003). Knowledge Sharing: A Review of Literature. Washington: The World Bank.

Daniel, W. (2002). Bioestadística: Base para el análisis de las ciencias de la salud. Cuarta edición. México: Limusa Wiley.

Delgado, M., Pedraja-Rejas, L. \& Rodríguez-Ponce, E. (2010). Estilos de Liderazgo y gestión de conocimiento en pequeñas empresas. Técnica Administrativa, 9(1) 1-13.

Eppard, R. (2004).Transformational and Transactional Leadership Styles as the Predict Constructive Culture and Defensive Culture. Blacksburg, Virginia. Polytechnic Institute and State University.

Ford, D. \& Staples, S. (2006). Perceived Value of Knowledge: The Potential Informer's Perception. Knowledge Management Research and Practice, 4, 3-16.

Gammelgaard, J., Husted, K., \& Michailova, S. (2004). Knowledge Sharing and Post-Acquisition Integration Failure. Working Paper 6, Copenhagen Business School, Denmark.

García-Morales, V., Llórens-Montes, F., \& Verdú-Jóver, A. (2008). The Effects of Transformational Leadership on Organizational Performance Through Knowledge and Innovation. British Journal of Management, 19, 299-319.

Hair, J., Anderson, R., Tatham, R. y Black, W. (2000). Análisis Mutivariante. Quinta edición. Madrid: Prentice Hall.

Hernández, R., Fernández, C., \& Baptista, P. (2006). Metodología de la Investigación. México D.F: Mc. Graw Hill.

Juárez, F., \& Contreras, F. (2012). Calidad de vida y liderazgo. Influencia de la calidad de vida percibida del directivo colombiano sobre sus prácticas de liderazgo. Acta Colombiana de Psicología, 15, 119-130.

Karkoulian, S., \& Masheredjian, J. (2012). Prediction of Knowledge Acquisition, Knowledge Sharing and Knowledge Utilization from Locus of Control: An Empirical Investigation. Business Studies Journal, 4, 117-130.

Lakshman, C. (2005). Top Executive Knowledge Leadership: Managing Knowledge to Lead Change at General Electric. Journal of Change Management, 5(4), 429-446.

Liao, L. (2006). A Learning Organization Perspective on Knowledge Sharing Behavior and Firm Innovation. Human Systems Management, 25, 227-236.

Liu, Y., \& DeFrank, R. (2013). Self-Interest and Knowledge Sharing Intentions: The Impact of Transformational
Leadership Climate and Human Resources Practices. International Journal of Human Resource Management, 24, 1151-1164.

Lu, L., Leung, P., \& Koch, P. (2006). Managerial Knowledge Sharing: The Role of Individual, Interpersonal and Organizational Factors. Management and Organization Review, 2, 15-41.

Lu, L., Leung, K., \& Tremain, P. (2006). Managerial Knowledge Sharing: The Role of Individual, Interpersonal, and Organizational Factors. Management and Organizational Review, 2(1), 15-41.

Maier, R., \& Schmidt, A. (2007). Characterizing Knowledge Maturing: A Conceptual Process Model for Integrating E-Learning and Knowledge Management. 4th Conference Professional Knowledge Management (WM 07), Postdam, Germany.

McDermott, R., \& O’Dell, C. (2001). Overcoming Cultural Barriers to Knowledge Sharing. Journal of Knowledge Management, 5, 76-85.

Mendoza, I. (2007). Perfil liderazgo transformacional de gerentes de ventas de una empresa químico-farmacéutica de clase mundial en México. XI Congreso Internacional de la Academia de Ciencias Administrativas, A.C., Jalisco, México.

Mendoza, M., \& Ortíz, C. (2006). El Liderazgo transformacional, dimensiones e impacto en la cultura organizacional y eficacia de las empresas. Revista Facultad de Ciencias Económicas. Investigación y Reflexión, 14(1) 118-134.

Nonaka, I., \& Takeuchi, H. (1995). La organización creadora de conocimiento: Cómo las empresas japonesas crean la dinámica de innovación. Oxford: Oxford University Press.

Palacios M., \& Garrigós, S. (2006). Propuesta de una escala de medida de la gestión del conocimiento en las industrias de biotecnología y telecomunicaciones. Investigaciones Europeas de Dirección y Economía de la Empresa, 12(1), 207-224.

Pauliene, R. (2012). Transforming leadership styles and knowledge sharing in multicultural context. Business, Management and Education, 10(1), 91-109.

Perrin, C., Perrin, B., Blauth, Ch., Apthorp, A., Duffy, R., Bonterre, M., \& Daniels, Sh. (2012). Factor analysis of global trends in twenty-first century leadership. Leadership \& Organizational Development Journal, 33(2), 175-199.

Prieto, G. \& Delgado, A. (2003). Análisis de un test mediante el modelo de Rasch. Psicothema, 15(1), 94-100.

Rodríguez-Ponce, E. (2007). Estilos de liderazgo, toma de decisiones estratégicas y eficacia: Un estudio empírico en pequeñas y medianas empresas. Interciencia, 32(8), 523528.

Sadler, P. (2003). Leadership and Organizational Learning. En Easterby-Smith, M. y Lyles, M. (2003). The Blackwell Handbook of Organizational Learning and Knowledge Management. Oxford: Blackwell Publishing. 
Salazar del Castillo J. (2004). Algunas reflexiones sobre la gestión del conocimiento en las empresas. Intangible Capital, 0(1), 1-6.

Shafieiyoun, S., \& Safaei, A. (2013). Enhancing Knowledge Sharing among Higher Education Students through Digital Game. Journal of Knowledge Management, Economics and Information Technology, 3(3), 1-20.

Shao, Z., Feng, Y., \& Liu, L. (2012). The Mediating Effect of Organizational Culture and Knowledge Sharing on Transformational Leadership and Enterprise Resource Planning Systems Success: An empirical study in China. Computers in Human Behavior, 28, 2400-2413.

Shih, H., Chiang Y., \& Chen, T. (2012). Transformational Leadership, Trusting Climate and Knowledge Exchange Behaviors in Taiwan. The International Journal of Human Resource Management, 23, 1057-1073.

Singh, S. (2008). Role of Leadership in Knowledge Management: A study. Journal of Knowledge Management, 12(4), 3-15.

Smith, G. (2009). Social Networking and the Transfer of Knowledge. The electronic Journal of Knowledge Management. 7(1), 165-178.
Song, J., Kolb, J., Lee, U., \& Kim, H. (2012). Role of Transformational Leadership in Effective Organizational Knowledge Creation Practices: Mediating Effects of Employees' Work Engagement. Human Resource Development Quarterly, 23, 65-101.

Sun, Z., \& Hao, G. (2006). HMS: A Hierarchical Spiral Model for Knowledge Management. Proceedings of the 2nd International Conference on Information Management and Business (IMB 2006), Sydney, Australia 13-16 February 2006, 542-551.

Vega, C., \& Zavala, G. (2004). Adaptación del cuestionario multifactorial de liderazgo (MLQ forma corta) de B. Bass y B. Avolio al contexto chileno. Universidad de Chile.

Yulk, G. (2009). Leading Organizational Learning: Reflections on Theory and Research. The Leadership Quarterly, 20, 49-53.

Zack, M. (2005). The Strategic Advantage of Knowledge and Learning. International Journal of Learning and Intellectual Capital, 2, 1-20. 